\title{
Geoquímica de las rocas plutónicas del batolito cambro-ordovícico del Cordón de Lila y de la Sierra de Almeida, Región de Antofagasta, Chile
}

\author{
Hans Niemeyer Rubilar ${ }^{1}$ \\ 1 Departamento de Ciencias Geológicas, Universidad Católica del Norte, Angamos 0610, Antofagasta, Chile. \\ hansn@ucn.cl
}

RESUMEN. En el Cordón de Lila y la Sierra de Almeida, región de Antofagasta, norte de Chile, afloran rocas plutónicas graníticas pertenecientes a un batolito cambro-ordovícico (490-460 Ma) que forma parte del arco magmático famatiniano. Los plutones que lo constituyen son de composición granodiorítica y monzogranítica, respectivamente. Los primeros ocupan una posición occidental en tanto que los segundos, oriental. La diferencia geoquímica entre ambos tipos de rocas es el enriquecimiento en sílice y $\mathrm{K}$ de las segundas respecto de las primeras. La mayoría de las granodioritas son metaluminosas o están en el límite entre el campo metaluminoso y el campo peraluminoso. Los monzogranitos son marcadamente peraluminosos. La composición química de los plutones (elementos mayores, en trazas y elementos de las tierras raras) permite inferir un origen común para ambos grupos, a partir de un magma máfico que evolucionó por cristalización fraccionada. Las diferencias de composición entre ambos, en particular su carácter meta- o peraluminoso, puede ser explicado por diferentes grados de interacción del magma con las rocas corticales félsicas.

Palabras clave: Batolito famatiniano, Geoquímica, Granodioritas, Monzogranitos.

\begin{abstract}
Cordón de Lila-Sierra de Almeyda Cambro-Ordovicic batholith: geochemistry of the plutonic rocks. Antofagasta region ,Chile. An Upper Cambrian-Ordovician (490-460 Ma) batholith crop out along the Cordón de Lila and Sierra de Almeida, in the Antofagasta region, northern Chile, which belongs to the Famatinian arc. Two plutonic rock types can be distinguished: granodiorites and monzogranites. The first lithology is positioned along an occidental fringe in as much the second is located along an oriental part of the batholith. The main geochemical difference between the two lithologies is the enrichment in silica and $\mathrm{K}$ of the second with respect of the first one. Most of the granodiorites are metaluminous or are located between the metaluminous and peraluminous fields. The monzogranites are mostly peraluminous. The chemical composition of the plutons (mayor and trace elements and rare earth elements) allow to infer a common origin for both groups, from a mafic magma that evolved by fractional crystallization. The difference in composition between both lithologies, specially the meta or peraluminous character, can be explained by means a different degree of interaction of the original magma with the felsic crustal rocks.
\end{abstract}




\section{Introducción}

El orógeno famatiniano representa un evento mayor desarrollado en el borde occidental de Gondwana en el Paleozoico temprano. El arco magmático emplazado durante este evento se extiende desde las sierras pampeanas en Argentina hasta el norte de los Andes en Venezuela (Ramos, 2018). En el área que comprende el Cordón de Lila (CL) y la Sierra de Almeida (SA), ubicada inmediatamente al sur del salar de Atacama, en el norte de Chile (Fig. 1), afloran rocas plutónicas graníticas pertenecientes a un batolito que forma parte del arco magmático famatiniano (Ducea et al., 2015), localmente denominado en Argentina Faja Eruptiva de la Puna Occidental (Palma et al., 1986), de orientación general noroeste. Este arco magmático se desarrolló sobre la corteza continental por subducción dirigida hacia el noreste (Bahlburg y Hervé, 1997), a lo largo del borde occidental de Gondwana durante el Cámbrico superior (Furongiano) a Ordovícico Medio (Zimmermann et al., 2010; Niemeyer et al., 2018), y abarcó un lapso de ca. 30 Ma entre 490 y $460 \mathrm{Ma}$ (Niemeyer et al., 2014).

El batolito que constituye el Cordón de Lila y la Sierra de Almeida presenta extensos afloramientos que han permitido su estudio detallado. Las características petrográficas de cada uno de los diferentes plutones que lo componen están bien establecidas y sus relaciones de contacto mutuas y con su roca de caja son claras y definidas (Mpodozis et al., 1983; Niemeyer, 2013). Dentro del batolito cambro-ordovícico (490-460 Ma) se pueden distinguir dos litologías principales de rocas plutónicas: granodioritas y monzogranitos (Fig. 2). El objetivo de la presente nota es dar a conocer el carácter geoquímico de los plutones que conforman dicho batolito, a la vez que sugerir ideas acerca de su origen.

\section{Marco geológico}

La roca de caja del batolito cambro-ordovícico del Cordón de Lila y de la Sierra de Almeida está formada por el Complejo ígneo-sedimentario del Cordón de Lila (CISL) (Niemeyer, 1989). Este se compone de dos miembros sub-contemporáneos a la edad de los plutones del batolito (490-460 Ma, Niemeyer et al., 2014). El miembro inferior, de ca. $2.500 \mathrm{~m}$ de espesor, está compuesto de lavas basálticas con niveles intercalados de turbiditas (Zimmermann et al., 2010). El miembro superior lo conforman unos $200 \mathrm{~m}$ de dacitas y brechas dacíticas, también con intercalaciones de turbiditas. Una edad ordovícica media ha sido asignada a este último, sobre la base de dos determinaciones U-Pb en zircones individuales (471 $\pm 6 \mathrm{Ma}$ y $468 \pm \mathrm{Ma}$ ) obtenidos en clastos de las brechas dacíticas (Niemeyer et al., 2018). Además, en la parte superior del Plutón Tambillo aflora un roof-pendant kilométrico de roca sedimentaria no turbidítica (Fig. 3), cuya edad es

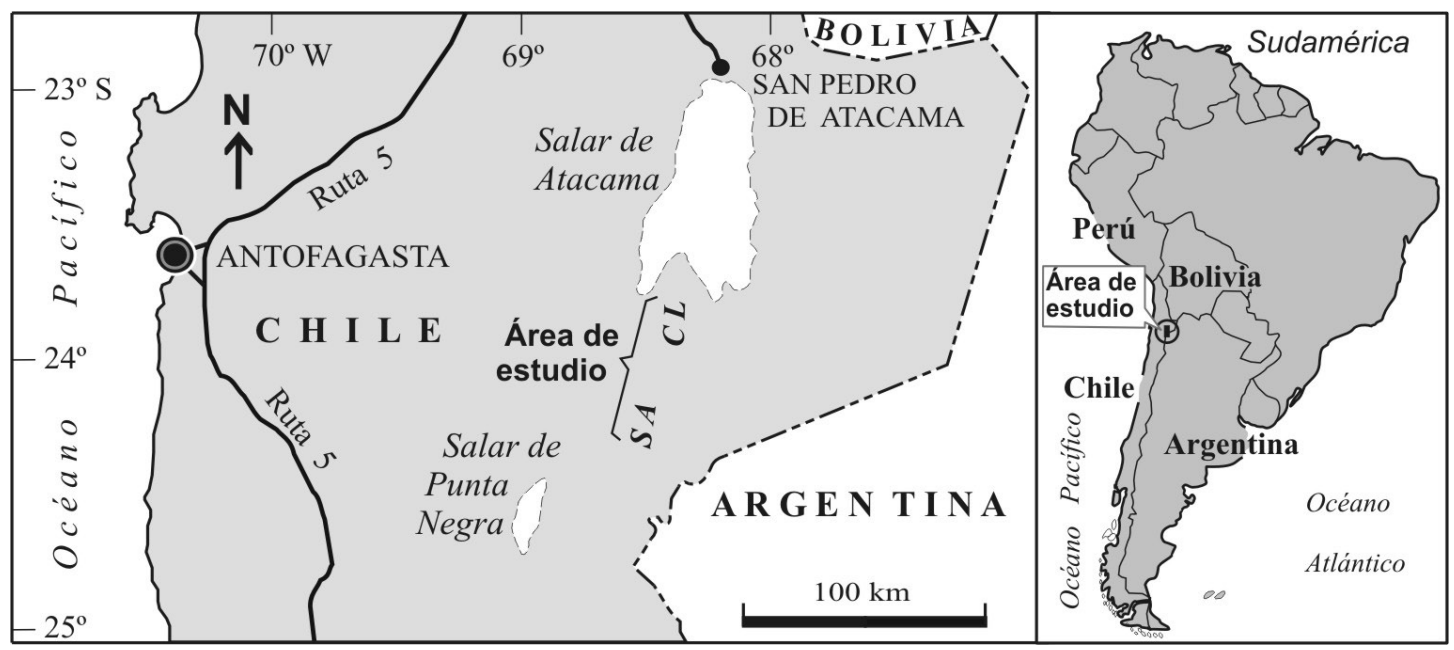

FIG. 1. Ubicación del área estudiada, en la precordillera de Antofagasta entre los salares de Atacama y Punta Negra. CL: Cordón de Lila, SA: Sierra de Almeida. 
(A)

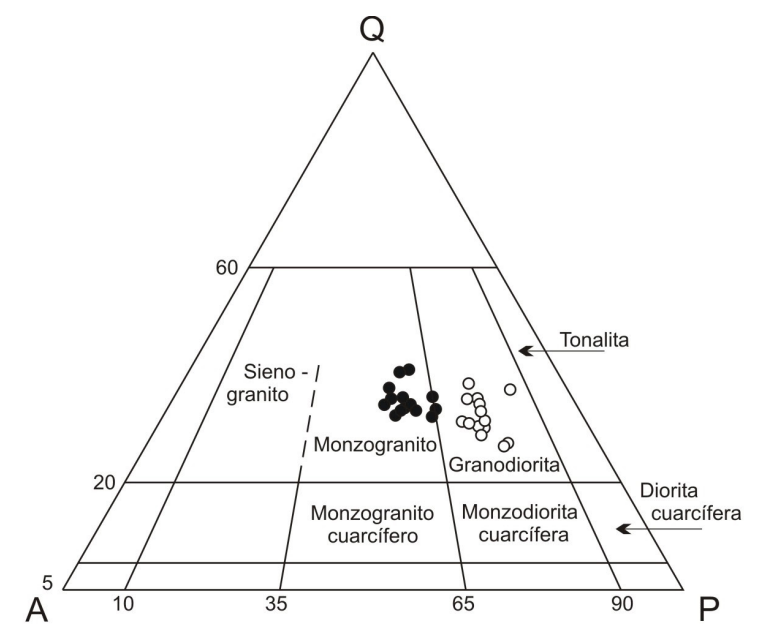

(B)

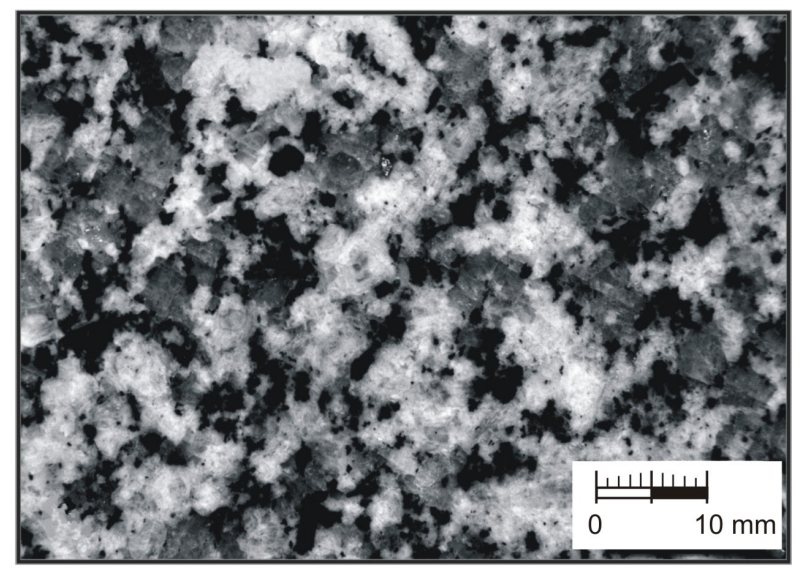

(C)

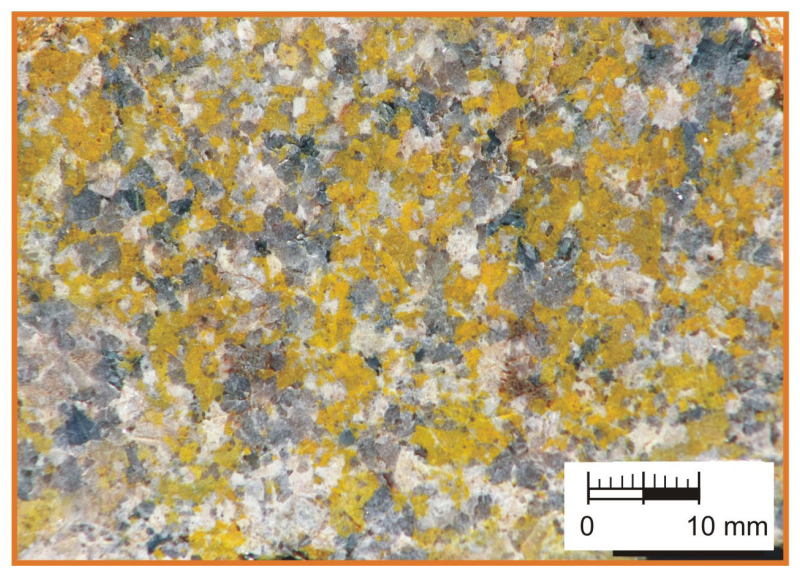

FIG. 2. A. Composición petrográfica de granodioritas y monzogranitos en diagrama QAP de Streckeisen (1974). Mineralogía según la norma C.I.P.W. B. Fotografía de una muestra granodiorítica del Plutón Pingo-Pingo, nótese la abundancia de biotita y anfíbola junto a cuarzo y plagioclasa. C. Fotografía de una muestra de monzogranito del Plutón Tilopozo en la que se ha teñido de amarillo el feldespato alcalino para destacar su abundancia. 


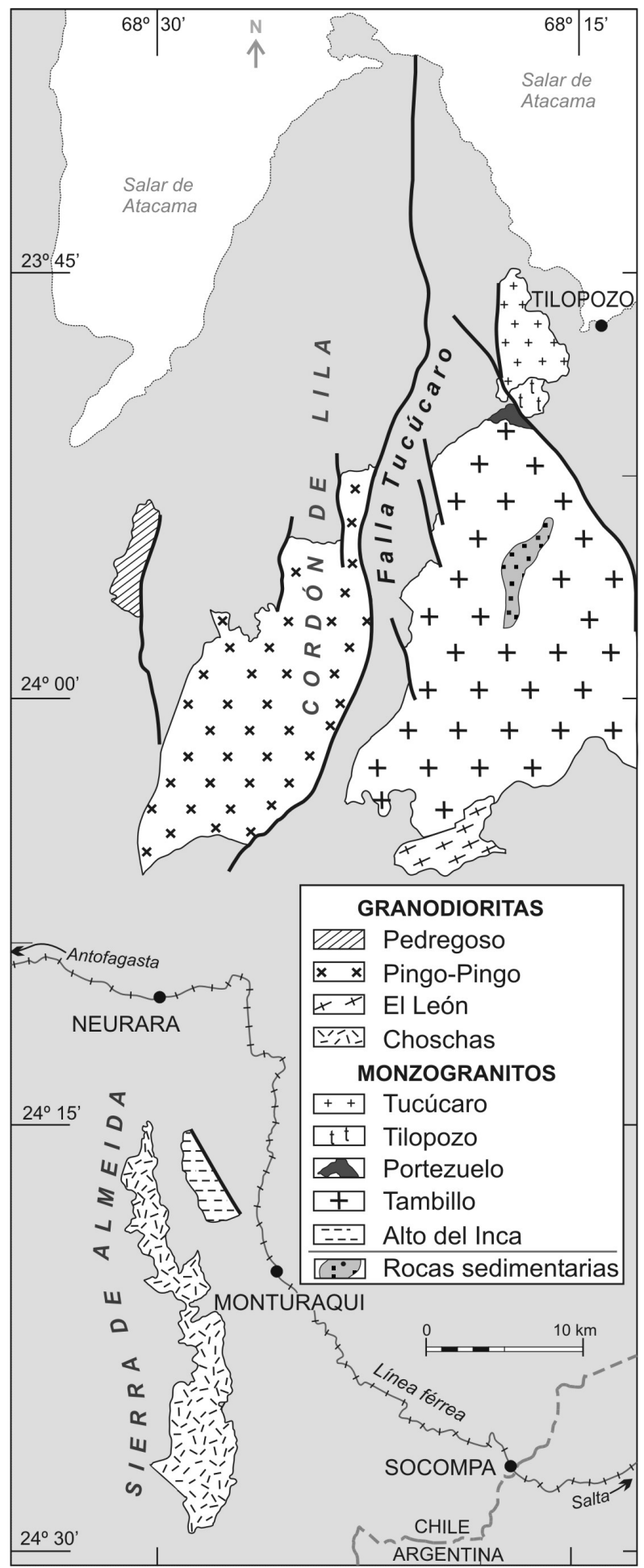

FIG. 3. Distribución de los plutones del batolito cambro-ordovícico del Cordón de Lila y Sierra de Almeida, al sur del salar de Atacama. Se distingue, en general, una composición granodiorítica en los plutones en posición occidental y una composición monzogranítica en los plutones en posición oriental. 
desconocida y que probablemente corresponda a un equivalente de la Formación Puncoviscana sensu lato, de edad proterozoica superior a cámbrica presente en Argentina (Aceñolaza y Aceñolaza, 2005).

El contacto de los diferentes plutones del batolito con su roca de caja es neto, con desarrollo en ellas de una aureola de rocas córneas de unos $50 \mathrm{~m}$ de ancho. Se caracterizan por haberse emplazado en la epizona a $<10 \mathrm{~km}$ de profundidad (Valencia, 2014). Los plutones son isótropos con excepción del Plutón El León, que presenta una marcada foliación magmática (Carrasco, 2015).

En general, los plutones granodioríticos (Pedregoso, Pingo-Pingo, El León y Choschas) ocupan una posición más bien occidental dentro del batolito, en tanto que los plutones monzograníticos (Tucúcaro, Tilopozo, Portezuelo, Tambillo y Alto del Inca) lo hacen en una posición oriental (Fig. 3).

La naturaleza del basamento del CISL se puede inferir a partir de los numerosos xenolitos de rocas metamórficas presentes en el Plutón El León y del roof-pendant alojado en el Plutón Tambillo. Los xenolitos son de tamaño centimétrico a decamétrico y se componen de micaesquistos, rocas sedimentarias calcosilicatadas y, en menor proporción y tamaño, anfibolitas. Estos xenolitos sugieren la presencia de un basamento de probable edad mesoproterozoica (Pankhurst et al., 2016).

\section{Metodología}

Se determinó la composición química de 28 muestras de roca de los diferentes plutones que conforman el batolito (Tablas 1 y 2). Los análisis químicos de elementos mayores y trazas fueron realizados en el Laboratorio Químico del Servicio Nacional de Geología y Minería, Santiago, Chile. Para ello se utilizó una combinación de técnicas analíticas y preparación de muestras que incluyen fusión y digestión ácida (elementos mayores y trazas) y determinación de los contenidos por espectrometría de acoplamiento inductivo con detector de masa (ICP-MS) y espectrometría de absorción atómica (EAA).

\section{Resultados}

El contenido de elementos mayores y en trazas determinado en las muestras analizadas se presenta en las tablas 1 y 2 para las granodioritas y los monzogranitos, respectivamente. La mayor parte de los valores de las determinaciones de pérdidas por calcinación (ppc) en cada muestra son menores al $1 \%$ en peso, lo cual es considerado como un indicador de que las muestras analizadas presentan un bajo grado de alteración, lo que permite inferir que su composición química no ha sufrido cambios significativos.

\subsection{Elementos mayores}

Las muestras de ambas litologías tienen un rango de variación en sus contenidos de $\mathrm{SiO}_{2}$ entre 65 y 77 peso \% con un aparente gap entre 73 y 74 peso \%. En los diagramas de Harker (Fig. 4) se advierte que, en general, en ambas litologías la variación de los elementos mayores con respecto a los contenidos de $\mathrm{SiO}_{2}$ definen patrones lineales de diferenciación, los cuales tienen la misma pendiente, especialmente el $\mathrm{MgO}_{2}, \mathrm{Fe}_{2} \mathrm{O}_{3}, \mathrm{Al}_{2} \mathrm{O}_{3}$ y $\mathrm{TiO}_{2}$. La variación de $\mathrm{CaO}$ con la sílice es también lineal en descenso con la misma pendiente para ambas litologías, con un gap de $\mathrm{CaO}$ en 2,38\% en las granodioritas y $1,31 \%$ de $\mathrm{CaO}$ en los monzogranitos (Fig. 4).

En el diagrama $\mathrm{Na}_{2} \mathrm{O}+\mathrm{K}_{2} \mathrm{O}-\mathrm{CaO}$ versus $\mathrm{SiO}_{2}$ (Frost et al., 2001), las granodioritas se distribuyen a lo largo de la serie cálcica y los monzogranitos lo hacen, en su mayoría, en el campo de la serie calcoalcalina y, parcialmente, en el campo de la serie álcali-cálcica. Sin embargo, parte de los análisis de monzogranitos caen hacia la derecha del diagrama, en un sector donde no hay división de campos (Fig. 5A).

Por otro lado, en el diagrama de $\mathrm{SiO}_{2}$ versus $\mathrm{K}_{2} \mathrm{O}$ (Rickwood, 1989) las granodioritas ocupan el campo normal de la serie calcoalcalina en tanto que los monzogranitos se distribuyen en el campo de la serie calcoalcalina rica en $\mathrm{K}$, aunque, dado su contenido de $\mathrm{SiO}_{2}$, la mayor parte de las muestras exceden hacia la derecha las divisiones establecidas por ese autor (Fig. 5B).

El diagrama molar de Shand (1943) de A/NKC versus $\mathrm{A} / \mathrm{NK}$ indica que la mayoría de las granodioritas son metaluminosas o están en el límite entre el campo metaluminoso y el peraluminoso, con una muestra en el campo de las rocas peraluminosas. La mayor parte de los monzogranitos son peraluminosos, con excepción de una muestra, que es metaluminosa (Fig. 5C). Adicionalmente, con la excepción del Plutón Tambillo, el resto de los plutones monzograníticos presentan corindón normativo $>1 \%$, lo cual significa un exceso de $\mathrm{Al}_{2} \mathrm{O}_{3}$ con respecto a $\mathrm{CaO}$. 
TABLA 1. ANÁLISIS QUÍMICOS DE GRANODIORITAS DEL BATOLITO DEL CORDÓN DE LILA Y LA SIERRA DE ALMEIDA.

\begin{tabular}{|c|c|c|c|c|c|c|c|c|c|c|c|c|c|c|}
\hline \multirow{2}{*}{\begin{tabular}{|l|} 
Plutón \\
Muestra
\end{tabular}} & \multicolumn{3}{|c|}{ Pedregoso } & \multicolumn{6}{|c|}{ Pingo-Pingo } & \multicolumn{4}{|c|}{ Choschas } & \multirow{2}{*}{$\begin{array}{c}\text { El León } \\
\text { TON }\end{array}$} \\
\hline & TON1 & TON2 & TON3 & PP02 & PP07 & PP15 & PP17 & PP19 & PP20 & CH07 & CH08 & CH10 & CH11 & \\
\hline $\mathrm{SiO}_{2} \%$ & 65,36 & 66,93 & 68,85 & 73,15 & 69,82 & 68,49 & 71,65 & 72,06 & 69,58 & 65,38 & 67,45 & 64,73 & 64,88 & 70,19 \\
\hline $\mathrm{TiO}_{2} \%$ & 0,42 & 0,41 & 0,3 & 0,21 & 0,28 & 0,38 & 0,25 & 0,2 & 0,27 & 0,47 & 0,36 & 0,44 & 0,46 & 0,42 \\
\hline $\mathrm{Al}_{2} \mathrm{O}_{3} \%$ & 14,92 & 15,12 & 14,78 & 14,3 & 14,66 & 14,51 & 14,87 & 13,47 & 13,96 & 15,45 & 15,54 & 16,14 & 15,28 & 14,38 \\
\hline $\mathrm{FeO} \%$ & 2,98 & 2,13 & 1,87 & 0,88 & 1,62 & 2,14 & 1,28 & 1,14 & 1,7 & 2,7 & 2,61 & 3,32 & 2,63 & 1,35 \\
\hline $\mathrm{Fe}_{2} \mathrm{O}_{3} \%$ & 2,33 & 2,45 & 1,72 & 1,29 & 1,97 & 2,03 & 1,07 & 1,47 & 1,84 & 2,71 & 1,75 & 2,06 & 2,59 & 1,55 \\
\hline $\mathrm{MnO} \%$ & 0,09 & 0,08 & 0,08 & 0,04 & 0,08 & 0,09 & 0,05 & 0,06 & 0,09 & 0,1 & 0,09 & 0,11 & 0,09 & 0,07 \\
\hline $\mathrm{MgO} \%$ & 2,23 & 1,5 & 1,18 & 0,44 & 0,9 & 1,34 & 0,58 & 1,14 & 1,7 & 1,87 & 1,37 & 1,8 & 2,38 & 1,52 \\
\hline $\mathrm{CaO} \%$ & 5,05 & 4,17 & 3,47 & 2,38 & 3,54 & 3,7 & 3,15 & 3,03 & 3,66 & 4,94 & 4,2 & 4,87 & 4,48 & 4,77 \\
\hline $\mathrm{Na}_{2} \mathrm{O} \%$ & 2,47 & 3,09 & 3,16 & 3,69 & 3,29 & 3,21 & 3,53 & 3,54 & 3,27 & 3,07 & 2,95 & 3 & 2,6 & 3,14 \\
\hline $\mathrm{K}_{2} \mathrm{O} \%$ & 2,55 & 2,54 & 3,01 & 2,41 & 2,39 & 3 & 2,47 & 2,65 & 2,47 & 2,15 & 2,51 & 2,29 & 2,58 & 1,49 \\
\hline $\mathrm{P}_{2} \mathrm{O}_{5} \%$ & 0,05 & 0,05 & 0,04 & 0,03 & 0,03 & 0,04 & 0,03 & 0,05 & 0,07 & 0,05 & 0,04 & 0,05 & 0,05 & 0,11 \\
\hline Рpc \% & 1,15 & 1,05 & 1,09 & 0,71 & 0,95 & 0,65 & 0,61 & 0,63 & 0,69 & 0,72 & 0,69 & 0,76 & 1,85 & 0,95 \\
\hline Total \% & 99,6 & 99,52 & 99,55 & 99,53 & 99,53 & 99,58 & 99,54 & 99,44 & 99,3 & 99,61 & 99,56 & 99,57 & 99,87 & 99,94 \\
\hline $\mathrm{Cu}$ ppm & 21 & 15 & 12 & 713 & 576 & 140 & 98 & 34 & $<5$ & 28 & 14 & 73 & 10 & 22 \\
\hline $\mathrm{V}$ ppm & 76 & 69 & 55 & 35 & 60 & 83 & 34 & 36 & 48 & 86 & 51 & 68 & 68 & 69 \\
\hline Cr ppm & 152 & 133 & 117 & 164 & 108 & 105 & 123 & 29 & 19 & 198 & 213 & 240 & 221 & 22 \\
\hline Co ppm & 15 & 12 & 11 & 7 & 11 & 13 & 8 & 6 & 6 & 15 & 13 & 15 & 15 & 9 \\
\hline Ni ppm & 62 & 62 & 55 & 57 & 55 & 57 & 57 & 12 & 9 & 56 & 56 & 58 & 70 & 11 \\
\hline Zn ppm & 44 & 73 & 64 & 604 & 541 & 168 & 99 & 39 & 47 & 69 & 59 & 95 & 54 & 49 \\
\hline $\mathrm{Rb}$ ppm & 76 & 77 & 106 & 76 & 79 & 105 & 99 & 54 & 46 & 79 & 86 & 72 & 80 & 33 \\
\hline Sr ppm & 241 & 260 & 364 & 165 & 171 & 162 & 243 & 43 & 65 & 150 & 100 & 111 & 184 & 272 \\
\hline Y ppm & 31 & 32 & 40 & $<10$ & $<10$ & 30 & 21 & 60 & 16 & 31 & 29 & 28 & 33 & 76 \\
\hline Zr ppm & 82 & 96 & 96 & 75 & 66 & 90 & 62 & 192 & 100 & 88 & 65 & 68 & 112 & 557 \\
\hline $\mathrm{Nb}$ ppm & 4 & 4 & 6 & 2 & 3 & 4 & 2 & 2 & 2 & 4 & 4 & 4 & 6 & 8 \\
\hline Ba ppm & 642 & 687 & 728 & 764 & 753 & 692 & 768 & 203 & 267 & 692 & 730 & 885 & 759 & 424 \\
\hline $\mathrm{Pb}$ ppm & $<10$ & $<10$ & 17 & 27 & 23 & $<10$ & $<10$ & $<5$ & 32 & 42 & 11 & 107 & 13 & 30 \\
\hline
\end{tabular}

\begin{tabular}{|c|c|c|c|c|c|c|c|c|c|c|c|c|c|c|}
\hline \multicolumn{15}{|c|}{ Tierras raras (ppm) } \\
\hline Plutón & La & $\mathrm{Ce}$ & $\mathrm{Pr}$ & Nd & Sm & $\mathbf{E u}$ & Gd & Tb & Dy & Ho & Er & Tm & $\mathbf{Y b}$ & Lu \\
\hline Pingo-Pingo & 24,29 & 48,8 & 5,23 & 19,72 & 3,76 & 0,68 & 3,86 & 0,7 & 4,25 & 0,9 & 2,8 & 0,42 & 2,83 & 0,45 \\
\hline Pedregoso & 29,98 & 62,53 & 5,84 & 20,99 & 3,56 & 0,62 & 3,03 & 0,5 & 3,08 & 0,62 & 1,93 & 0,31 & 2,1 & 0,29 \\
\hline Choschas & 15,22 & 34,01 & 3,51 & 13,27 & 2,45 & 0,65 & 2,64 & 0,46 & 2,77 & 0,59 & 1,78 & 0,28 & 1,98 & 0,27 \\
\hline El León & 12,47 & 26,68 & 3,15 & 14,26 & 2,86 & 1,19 & 3,07 & 0,57 & 3,46 & 0,73 & 2,21 & 0,31 & 2,04 & 0,36 \\
\hline
\end{tabular}




\begin{tabular}{|c|c|c|c|c|c|c|c|c|c|c|c|c|c|c|}
\hline \multirow{2}{*}{$\begin{array}{c}\text { Plutón } \\
\text { Muestra }\end{array}$} & \multicolumn{4}{|c|}{ Tucúcaro } & \multicolumn{2}{|c|}{ Tilopozo } & \multirow{2}{*}{$\begin{array}{c}\text { Portezuelo } \\
\text { GRG1 } \\
\end{array}$} & \multicolumn{6}{|c|}{ Tambillo } & \multirow{2}{*}{$\begin{array}{c}\begin{array}{c}\text { Alto del } \\
\text { Inca }\end{array} \\
\text { AI-1 } \\
\end{array}$} \\
\hline & MC02 & MC05 & MC19 & MC09 & MC19 & Tilo 1 & & 6.2 & 7.1 & 10.3 & 10.7 & 10.1 & 11.3 & \\
\hline $\mathrm{SiO}_{2} \%$ & 74,95 & 74,72 & 74,67 & 76,4 & 74,91 & 75,31 & 77,02 & 76,09 & 75,59 & 76,77 & 74,46 & 74,5 & 74,83 & 76,72 \\
\hline $\mathrm{TiO}_{2}^{2} \%$ & 0,1 & 0,09 & 0,09 & 0,07 & 0,08 & 0,11 & $<0,02$ & 0,07 & 0,15 & 0,05 & 0,17 & 0,13 & 0,12 & 0,14 \\
\hline $\mathrm{Al}_{2} \mathrm{O}_{3} \%$ & 13,79 & 13,94 & 13,28 & 13,1 & 13,83 & 13,93 & 12,22 & 12,99 & 13,02 & 12,7 & 13,32 & 13,2 & 13,21 & 12,42 \\
\hline $\mathrm{FeO} \%$ & 0,58 & 0,54 & 0,57 & 0,37 & 0,27 & 0,24 & 0,84 & 0,22 & 0,38 & 0,08 & 0,88 & 0,66 & 0,85 & 1,04 \\
\hline $\mathrm{Fe}_{2} \mathrm{O}_{3} \%$ & 0,91 & 0,89 & 0,96 & 0,25 & 0,39 & 0,44 & 0,33 & 0,52 & 0,61 & 0,38 & 0,64 & 0,51 & 0,13 & 0,2 \\
\hline $\mathrm{MnO} \%$ & 0,05 & 0,05 & 0,06 & 0,03 & 0,06 & 0,06 & 0,06 & 0,06 & 0,04 & 0,04 & 0,03 & 0,08 & 0,06 & 0,04 \\
\hline $\mathrm{MgO} \%$ & 0,15 & 0,15 & 0,16 & 0,06 & 0,03 & 0,06 & 0,06 & 0,04 & 0,04 & 0,03 & 0,08 & 0,06 & 0,06 & 0,04 \\
\hline $\mathrm{CaO} \%$ & 0,87 & 0,9 & 1,1 & 0,61 & 0,5 & 0,66 & 0,27 & 0,59 & 1,18 & 0,38 & 1,15 & 1,31 & 1,21 & 0,37 \\
\hline $\mathrm{Na}_{2} \mathrm{O} \%$ & 4,13 & 4,36 & 4,34 & 3,54 & 3,88 & 3,52 & 3,62 & 4,24 & 3,78 & 3,86 & 3,5 & 3,62 & 3,73 & 3,66 \\
\hline $\mathrm{K}_{2} \mathrm{O} \%$ & 3,66 & 3,79 & 3,89 & 4,71 & 4,97 & 5,1 & 4,18 & 4,41 & 4,5 & 4,87 & 4,41 & 4,75 & 4,58 & 3,92 \\
\hline $\mathrm{P}_{2} \mathrm{O}_{5} \%$ & 0,02 & 0,01 & 0,02 & 0,01 & 0,01 & 0,02 & 0,03 & 0,08 & 0,07 & 0,06 & 0,08 & 0,06 & 0,06 & 0,02 \\
\hline ppc $\%$ & 0,46 & 0,47 & 0,44 & 0,78 & 0,51 & 0,5 & 0,97 & 0,23 & 0,37 & 0,24 & 0,74 & 0,51 & 0,54 & 1,23 \\
\hline Total \% & 99,67 & 99,91 & 99,58 & 99,93 & 99,51 & 99,97 & 99,71 & 99,66 & 99,93 & 99,47 & 99,96 & 99,61 & 99,57 & 99,84 \\
\hline $\mathrm{Cu}$ ppm & 19 & 12 & 18 & 12 & 18 & 16 & $<5$ & 7 & 11 & 7 & 12 & 6 & 7 & $<5$ \\
\hline $\mathrm{V}$ ppm & 9 & 10 & 7 & 1 & 7 & 5 & $<10$ & 7 & 12 & 6 & 25 & 10 & 7 & $<10$ \\
\hline Cr ppm & 103 & 128 & 125 & 207 & 125 & 119 & 48 & 295 & 315 & 277 & 332 & 272 & 240 & 14 \\
\hline Co ppm & 6 & 6 & 6 & 4 & 6 & 4 & $<5$ & 0 & 0 & 0 & 0 & 0 & 0 & $<5$ \\
\hline Ni ppm & 55 & 54 & 56 & 57 & 56 & 57 & $<5$ & 2 & 3 & 4 & 3 & 4 & 4 & $<5$ \\
\hline Zn ppm & 69 & 59 & 57 & $<5$ & 57 & 4 & 29 & 8 & 21 & 8 & 85 & 30 & 46 & 30 \\
\hline $\mathrm{Rb} p p m$ & 131 & 135 & 130 & 307 & 130 & 263 & 224 & 179 & 135 & 204 & 148 & 134 & 133 & 134 \\
\hline Sr ppm & 31 & 23 & 22 & $<5$ & 22 & $<5$ & $<5$ & 45 & 55 & 21 & 68 & 76 & 79 & $<5$ \\
\hline Y ppm & 62 & 10 & 61 & 104 & 61 & 83 & 52 & 45 & 31 & 43 & 37 & 28 & 28 & 28 \\
\hline $\mathrm{Zr} \mathrm{ppm}$ & 61 & 54 & 46 & 18 & 46 & 17 & 72 & 78 & 99 & 81 & 100 & 97 & 89 & 101 \\
\hline $\mathrm{Nb}$ ppm & 5 & 6 & 7 & 16 & 7 & 10 & 23 & 6 & 2 & 14 & 5 & 3 & 3 & 5 \\
\hline Ba ppm & 1.000 & 982 & 1.010 & 674 & 1.010 & 674 & 240 & 264 & 552 & 246 & 368 & 635 & 588 & 965 \\
\hline $\mathrm{Pb} \mathrm{ppm}$ & 58 & 50 & 60 & 38 & 60 & 62 & 28 & 13 & 14 & 23 & 33 & 24 & 22 & 23 \\
\hline \multicolumn{15}{|c|}{ Tierras raras (ppm) } \\
\hline Plutón & $\mathrm{La}$ & $\mathrm{Ce}$ & $\operatorname{Pr}$ & $\mathrm{Nd}$ & $\mathrm{Sm}$ & $\mathrm{Eu}$ & $\mathrm{Gd}$ & $\mathrm{Tb}$ & Dy & Ho & Er & $\mathrm{Tm}$ & $\mathrm{Yb}$ & $\mathrm{Lu}$ \\
\hline Tilopozo & 14,81 & 48,78 & 6,64 & 26,04 & 7,36 & 0,12 & 5,34 & 0,76 & 4,02 & 0,69 & 1,87 & 0,35 & 2,59 & 0,43 \\
\hline Tucúcaro & 25,53 & 63,08 & 6,79 & 27,71 & 5,87 & 0,83 & 6,84 & 1,25 & 7,42 & 1,52 & 4,39 & 0,71 & 4,78 & 0,66 \\
\hline Portezuelo & 21,3 & 50,42 & 5,82 & 20,68 & 5,12 & 0,27 & 3,9 & 0,66 & 3,84 & 0,82 & 2,32 & 0,41 & 2,77 & 0,46 \\
\hline Tambillo & 24,68 & 51,15 & 5,42 & 17,85 & 3,2 & 0,6 & 2,83 & 0,46 & 2,83 & 0,55 & 2 & 0,27 & 1,94 & 0,33 \\
\hline Alto del Inca & 25,1 & 56,05 & 5,83 & 20,84 & 3,96 & 0,89 & 2,54 & 0,38 & 1,76 & 0,34 & 1,04 & 0,16 & 0,87 & 0,21 \\
\hline
\end{tabular}




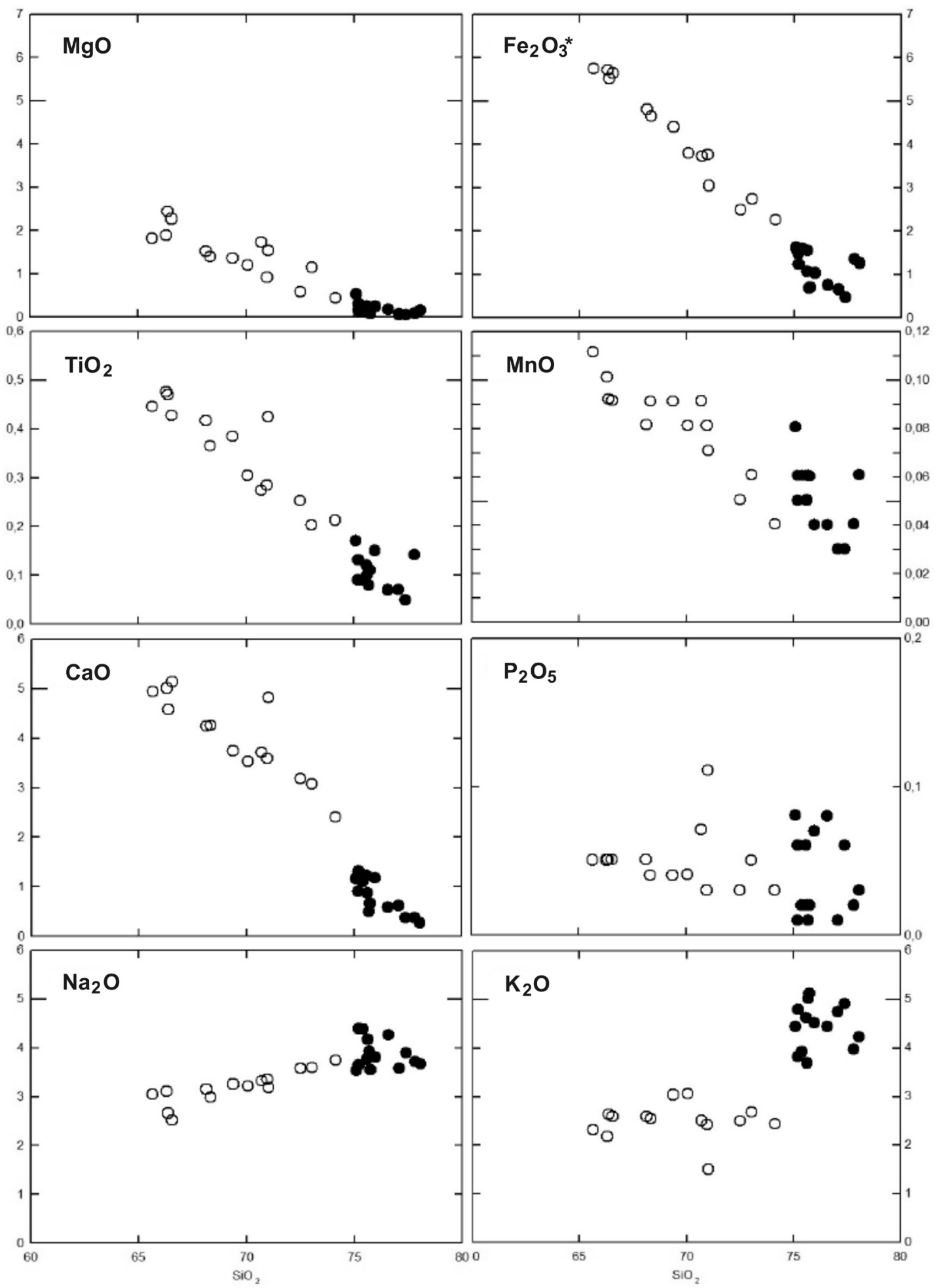

FIG. 4. Diagramas de Harker para elementos mayores de granodioritas (círculos vacíos) y de monzogranitos (círculos llenos), contenidos en peso $\%$. 
(A)

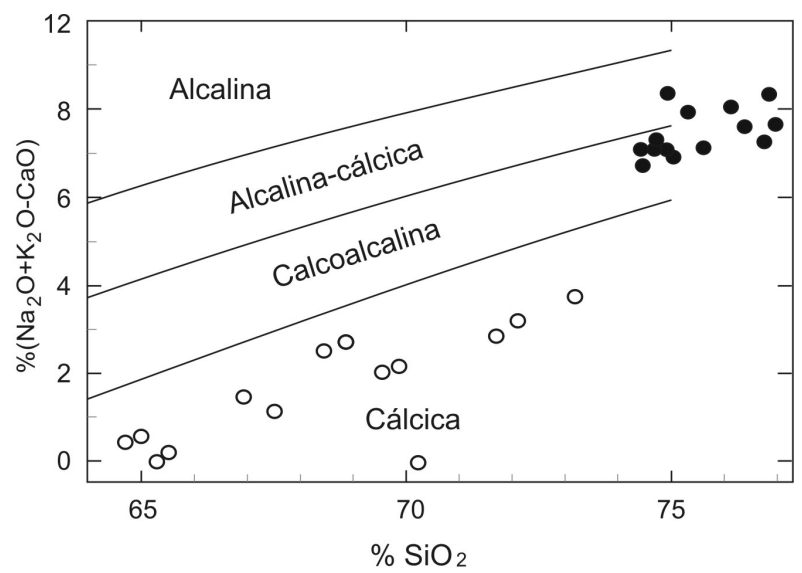

(B)

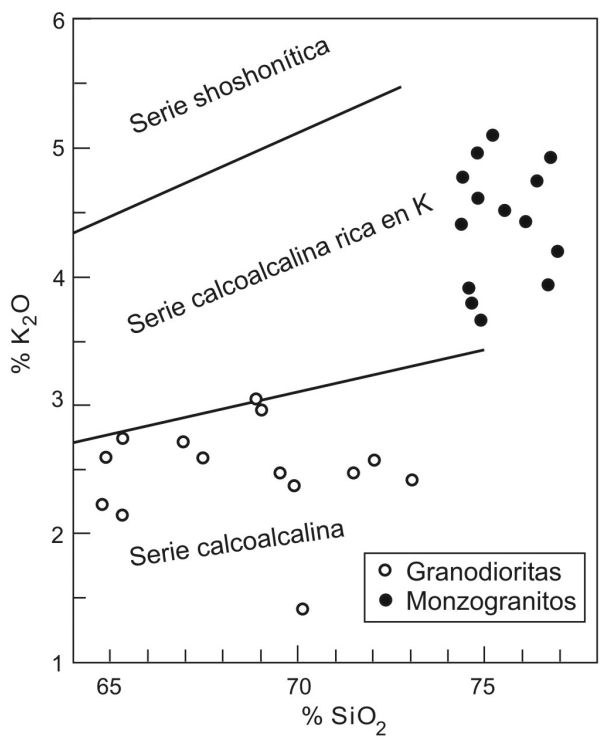

(C)

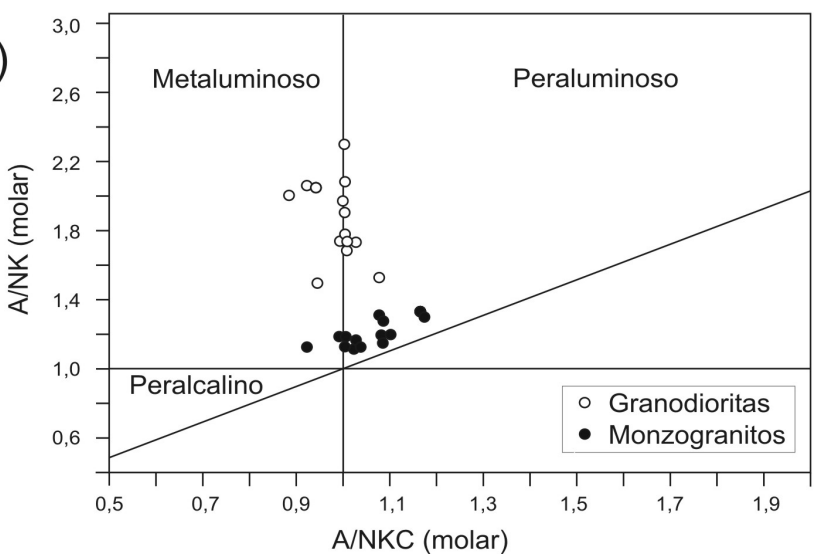

FIG. 5. A. Diagrama $\mathrm{Na}_{2} \mathrm{O}+\mathrm{K}_{2} \mathrm{O}-\mathrm{CaO}$ versus $\mathrm{SiO}_{2}$ de Frost et al. (2001) para las granodioritas (círculos vacíos) y para los monzogranitos (círculos llenos), contenidos en peso \%. B. Distribución de las granodioritas (círculos vacíos) y de los monzogranitos (círculos llenos) en el diagrama de $\mathrm{SiO}_{2}$ versus $\mathrm{K}_{2} \mathrm{O}$ (Rickwood, 1989), contenidos en peso \%. C. Diagrama de Shand (1943), A/NKC versus $\mathrm{A} / \mathrm{NK}$, para granodioritas y monzogranitos. La división transversal es tomada de Maniar y Piccoli (1989). 


\subsection{Elementos en trazas}

Se observa una diferencia notable entre los contenidos de los elementos en trazas de ambas litologías. En la figura 6 se indican las variaciones de algunos de estos elementos versus el contenido en $\mathrm{SiO}_{2}$. El Rb se enriquece en forma hiperbólica con el incremento del contenido de $\mathrm{SiO}_{2}$, desde las granodioritas hacia los monzogranitos (Fig. 6). El Sr de las granodioritas tiene una amplia dispersión, pero de modo general muestra una tendencia descendente con el incremento del contenido de $\mathrm{SiO}_{2}$. Para los monzogranitos, el $\mathrm{Sr}$ desciende linealmente dentro de su estrecho contenido de sílice (Fig. 5). La distribución de $\mathrm{Nb}$ es ligeramente descendente con el incremento de $\mathrm{SiO}_{2}$ para las granodioritas, y en los monzogranitos asciende linealmente entre $1 \mathrm{y}$ 24 ppm dentro de su estrecho rango del contenido de $\mathrm{SiO}_{2}$ (Fig. 6).

Los únicos plutones para los cuales se dispone de razones iniciales de ${ }^{87} \mathrm{Sr} r{ }^{86} \mathrm{Sr}$ son el Plutón Tucúcaro $(0,7102)$ y el Plutón Tilopozo (0,7113), (Mpodozis et a., 1983).
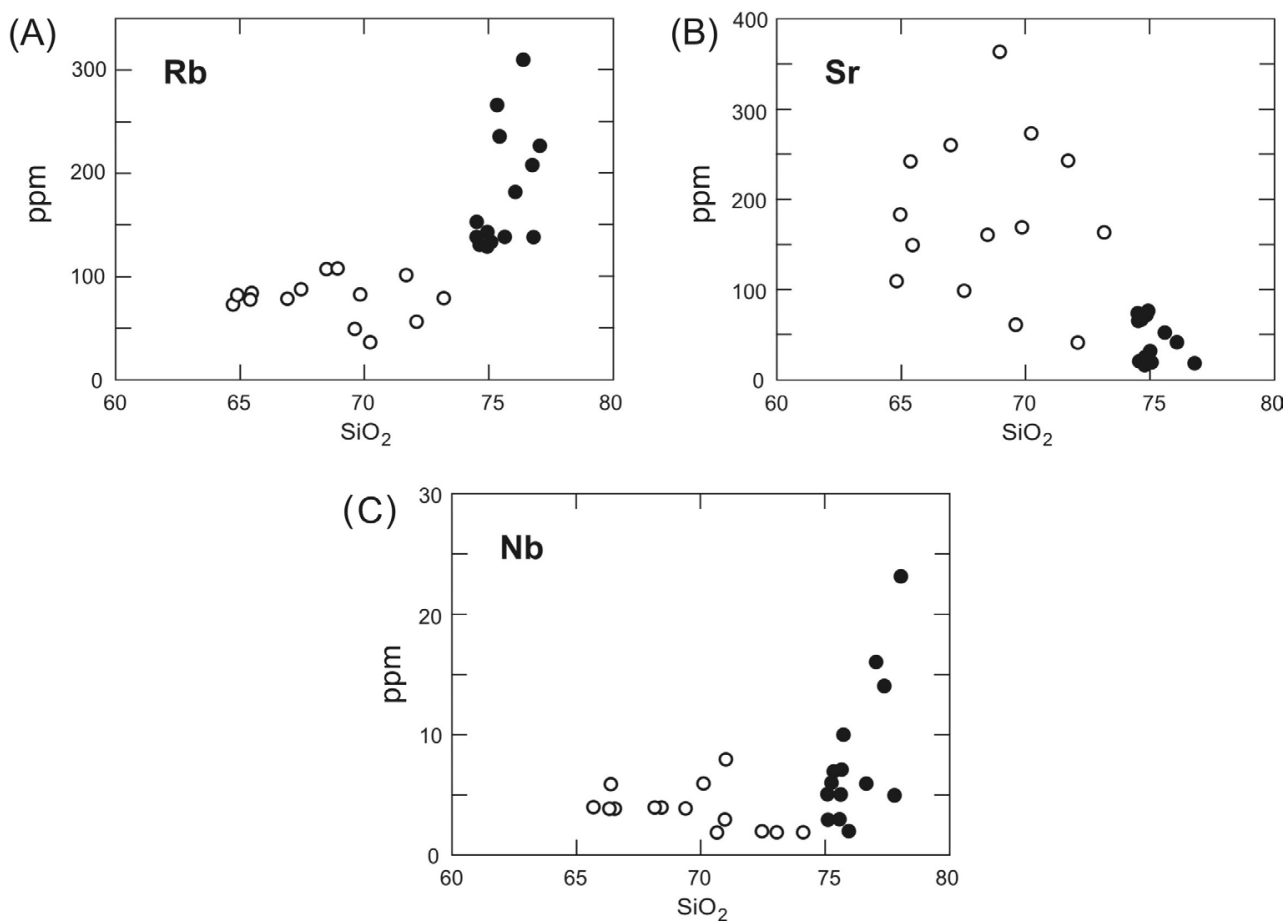

FIG. 6. Diagramas de Harker correspondientes a algunos elementos en trazas, contenidos en ppm. Granodioritas (círculos vacíos) y monzogranitos (círculos llenos), contenidos de $\mathrm{SiO}_{2}$ en peso\%. 

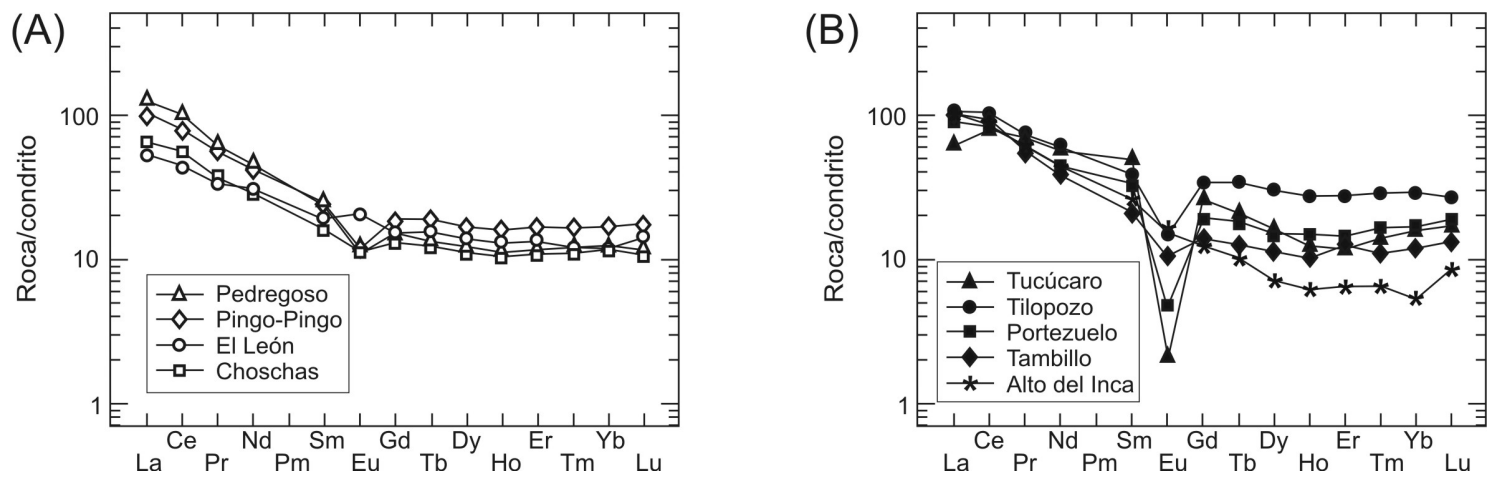

FIG. 7. A. Diagrama de tierras raras para granodioritas. B. Diagrama de tierras raras para monzogranitos. Contenidos de los elementos de las tierras raras normalizados a condrito (Boynton, 1984).

sierras pampeanas occidentales, noroeste de Argentina. Dicho modelo plantea que las rocas plutónicas calcoalcalinas del arco se habrían generado mediante procesos de cristalización fraccionada a partir de un magma básico, acompañados por procesos de contaminación/asimilación de metasedimentitas pertenecientes a la Formación Puncoviscana y/o interacción con fundidos félsicos originados en la corteza. Este modelo explica, en particular, el hecho de que las granodioritas y las monzonitas presentan un trend composicional que atraviesa el límite entre los campos metaluminoso y peraluminoso, por lo cual la explicación más simple para la generación de dichas secuencias es la interacción entre magmas de composición calcoalcalina y fundidos corticales o rocas de la corteza sedimentaria (Otamendi et al., 2012).

Este modelo petrogenético es aplicable a las rocas estudiadas en el presente trabajo, dado que las granodioritas se distribuyen en el campo metaluminoso con transición al campo peraluminoso en el diagrama de Shand (1943) (Fig. 5C). En cuanto a los monzogranitos, su carácter peraluminoso, unido a las altas razones iniciales de ${ }^{87} \mathrm{Sr} /{ }^{86} \mathrm{Sr}$, sugieren una contaminación con rocas sedimentarias corticales posiblemente representadas por el roof-pendant observado en el Plutón Tambillo y que serían un equivalente de la Formación Puncoviscana, la cual constituye el basamento sobre el que se emplazó el arco magmático famatiniano (Niemeyer, 2013).

Las granodioritas y los monzogranitos objeto de este estudio parecen haberse originado a partir de un magma calcoalcalino común que evolucionó a través de un proceso de cristalización fraccionada. Este origen común es respaldado por el comportamiento de los elementos mayores y en trazas en los diagramas de Harker (Figs. 4 y 6) y por los patrones normalizados de los elementos de las tierras raras (Fig. 7). Estos son compatibles con una evolución por cristalización fraccionada de plagioclasa de los magmas. La pronunciada anomalía de Eu observada en las muestras de los monzogranitos refuerza esta sugerencia (Fig. 7). En efecto, la cristalización temprana de plagioclasa, por ejemplo en las granodioritas, contribuye a secuestrar el Eu del líquido residual, lo que explica la anomalía negativa en los monzogranitos y el predominio de feldespato potásico sobre plagioclasa en esta litología (Figs. 2 y 7) (Cullers y Graf, 1984). Lo anterior es también compatible con el descenso del contenido de $\mathrm{CaO}$ y $\mathrm{Sr}$, con el incremento de $\mathrm{SiO}_{2}$ observado en los diagramas de Harker, y con la abundante cristalización de feldespato potásico en los monzogranitos. El cambio de comportamiento de todos estos elementos durante el proceso de cristalización sugiere un enriquecimiento de los elementos incompatibles, por ejemplo $\mathrm{K}_{2} \mathrm{O}, \mathrm{Rb}$ y Nb en el líquido residual (e.g., Rollinson, 1993; Lee y Morton, 2015), los cuales habrían pasado a formar parte de los monzogranitos.

\section{Conclusión}

El batolito del Cordón de Lila y Sierra de Almeida, ubicado al sur del salar de Atacama en el norte de Chile, está constituido por una serie de plutones de composición granodiorítica y monzogranítica, emplazados en una corteza siálica (principalmente sedimentaria) durante el Cámbrico Superior- Ordovícico. 
Ellos forman parte del arco magmático famatiniano y representan su borde occidental.

La composición química de los plutones (elementos mayores, en trazas y tierras raras) sugiere que tanto los plutones granodioríticos como los monzograníticos se formaron por cristalización fraccionada a partir de un magma máfico común. El carácter metaluminoso de las granodioritas y peraluminoso de los monzogranitos puede ser explicado por diferentes grados de interacción del magma con las rocas corticales sedimentarias.

\section{Agradecimientos}

Se agradece la revisión crítica del manuscrito por parte de L. Baeza, V. Oliveros, J. Muñoz, I. Urraza y W. Vivallo. Los dibujos se deben a L. Jofré, de la Universidad Católica del Norte.

\section{Referencias}

Aceñolaza, F.; Aceñolaza, G. 2005. La Formación Puncoviscana y unidades estratigráficas vinculadas en el Neoproterozoico-Cámbrico temprano del Noroeste Argentino. Latin American Journal of Sedimentology and Basin Analysis 12 (2): 65-87.

Bahlburg, H.; Hervé, F. 1997. Geodynamic evolution and tectonostratigraphic terranes of northwestern Argentina and northern Chile. Geological Society of America Bulletin 109 (7): 869-884. doi:10.1130/00167606(1997)109<0869:GEATTO>2.3.CO;2.

Boynton, W.V. 1984. Geochemistry of the rare earth elements: meteorite studies. In Rare earth element geochemistry (Henderson, P.; editor). Elsevier: 63-114.

Carrasco, J.L. 2015. La Granodiorita El León, extremo sureste del Cordón de Lila, Región de Antofagasta, Chile. Memoria de título (Inédita), Universidad Católica del Norte: 52 p.

Cullers, R.L.; Graf, J.L. 1984. Rare Earth elements in igneous rocks of the continental crust: intermediate and silicic rocks-ore petrogenesis. In Rare Earth Element Geochemistry (Henderson, P.; editor). Elsevier: 275-316.

Ducea, M.N.; Otamendi, J.E.; Bergantz, G.W.; Jianu, D.; Petrescu, L. 2015. The origin and petrologic evolution of the Ordovician Famatinian-Puna arc. In Geodynamics of a Cordilleran Orogenic System: The Central Andes of Argentina and Northern Chile (DeCelles, P.G.; Ducea, M.N.; Carrapa, B.; Kapp, P.A.; editores). Geological Society of America Memoir 212: 125-138.
Frost, B.R.; Barnes, C.G.; Collins, W.J.; Arculus, R.J.; Ellis, D.J.; Frost, C.D. 2001. A Geochemical Classification for Granitic Rocks. Journal of Petrology 42 (11): 2033-2048.

Lee, C.T.A.; Morton, D.M. 2015. High silica granites: Terminal porosity and crystal settling in shallow magma chambers. Earth and Planetary Science Letters 409: 23-31. doi: 10.1016/j.eps1.2014.10.040.

Maniar, P.D.; Piccoli, P.M. 1989. Tectonic discrimination of granitoids. Geological Society of America Bulletin 101 (5): 635-643. doi: 10.1130/0016-7606(1989)101<0635:TD $\mathrm{OG}>2.3 . \mathrm{CO} ; 2$.

Mpodozis, C.; Hervé, F.; Davidson, J.; Rivano, S. 1983. Los granitoides de Cerro Lila, manifestaciones de un episodio intrusivo y termal del Paleozoico inferior en los Andes del Norte de Chile. Revista Geológica de Chile 18: 3-14. doi: 10.5027/andgeoV10n1-a01.

Niemeyer, H. 1989. El Complejo ígneo-sedimentario del Cordón de Lila, Región de Antofagasta: significado tectónico. Revista Geológica de Chile 16 (2): 163 181. doi: 10.5027/andgeoV16n2-a03.

Niemeyer, H. 2013. Geología del Área Cerro Lila-Peine, Región de Antofagasta. Servicio Nacional de Geología y Minería, Carta Geológica de Chile, Serie Geología Básica 147: 37 p., 1 mapa escala 1: 100.000.

Niemeyer, H.; Meffre, S.; Guerrero, R. 2014. Zircon U-Pb geochronology of granitic rocks of the Cordón de Lila and Sierra de Almeida ranges, northern Chile: 30 m.y. of Ordovician plutonism on the western border of Gondwana. Journal of South American Earth Sciences 56: 228-241. doi: 10.1016/j.jsames.2014.09.011.

Niemeyer, H.; Götze, J.; Sanhueza, M.; Portilla, C. 2018. The Ordovician magmatic arc in the northern ChileArgentina Andes between $21^{\circ}$ and $26^{\circ}$ south latitud. Journal of South American Earth Sciences 81: 204214. doi: 10.1016/j.jsames.2017.11.016.

Otamendi, J.E.; Pinotti, L.P.; Basei, M.A.S.; Tibaldi, A.M. 2010. Evaluation of petrogenetic models for intermediate and silicic plutonic rocks from the Sierra de Valle Fértil-La Huerta, Argentina: Petrologic constraints on the origin of igneous rocks in the Ordovician Famatinian- Puna paleoarc. Journal of South American Earth Sciences 30: 29-45. doi: 10.1016/j.jsames.2010.07.004.

Otamendi, J.E.; Ducea, M.N.; Bergantz, G.W. 2012. Geological, Petrological and Geochemical Evidence for Progressive Construction of an Arc Crust Section, Sierra de Valle Fértil, Famatinian Arc, Argentina. Journal of Petrology 53 (4): 761-800. doi: 10.1093/ petrology/egr079. 
Palma, M.A.; Parica, P.D.; Ramos, V.A. 1986. El Granito Archibarca: su edad y significado tectónico, Provincia de Catamarca. Revista de la Asociación Geológica Argentina XLI (3-4): 414-419.

Pankhurst, R.J.; Hervé, F.; Fanning, C.M.; Calderón, M.; Niemeyer, H.; Griem-Klee, S.; Soto, F. 2016. The preMesozoic rocks of northern Chile: $\mathrm{U}-\mathrm{Pb}$ ages, and $\mathrm{Hf}$ and O isotopes. Earth Science Reviews 152: 88-105. doi: 10.1016/j.earscirev.2015.11.009.

Ramos, V. 2018. The Famatinian Orogen along the protomargin of western Gondwana: evidence of nearly continuous Ordovician magmatic arc between Venezuela and Argentina. In The evolution of the Chilean-Argentinean Andes (Folguera, A.; ContrerasReyes, E.; Neredia, N.; Encinas, A.; Iannelli, S.B.; Oliveros, V.; Dávila, F.M.; Collo, G.; Giambiagi, L.; Maksymowicz, A.; Iglesia Llanos, M.P.; Turienzo, M.; Naipauer, M.; Orts, D.; Litvak, V.D.; Álvarez, O.; Arriagada, C.; editors). Springer: 133-161.

Rickwood, P.C. 1989. Boundary lines within petrologic diagrams which use oxides of major and minor elements. Lithos 22 (4): 247-263. doi: 10.1016/00244937(89)90028-5.
Rollinson, H. 1993. Using geochemical data: evaluation, presentation, interpretation. Pearson, Prentice Hall: $352 \mathrm{p}$.

Shand, S. 1943. Eruptive Rocks: their genesis, composition, and classification, with a chapter on meteorites. John Wiley and Sons: 444 p.

Streckeisen, A. 1974. Rocas plutónicas. Clasificación y nomenclatura recomendada por la Unión Internacional de Ciencias Geológicas (I.U.G.S.). Subcomisión en sistemática de rocas ígneas. Traducido al Castellano de Neues Jahrbuch für Mineralogie, Abhandlungen, Instituto de Investigaciones Geológicas 107: 144240. Santiago.

Valencia, E. 2014. Caracterización geológico-estructural del borde norte del Plutón Pingo-Pingo y su aureola de contacto, Cordón de Lila, Región de Antofagasta. Memoria de título (Inédito), Universidad Católica del Norte: $134 \mathrm{p}$.

Zimmermann, U.; Niemeyer, H.; Meffre, S. 2010. Revealing the continental margin of Gondwana: the Ordovician arc of the Cordón de Lila (northern Chile). International Journal of Earth Sciences 99: 39-56. doi: 10.1007/s00531-009-0483-8.

Manuscript received: October 11, 2019; revised/accepted: July 7, 2020; available online: July 7, 2020. 